\title{
Women's Health Initiative: complex answers to simple questions on dietary fat and breast cancer
}

Susan T Mayne

The Women's Health Initiative (WHI) set out to answer the following question: does reducing dietary fat lower the incidence of breast cancer? ${ }^{1}$ It seemed like a straightforward question. The hypothesis that dietary fat might be related to breast cancer risk was based on compelling evidence from rodent mammary carcinogenesis models, geographic differences in breast cancer risk, migrant and time-trends studies, and case-control studies. $^{2,3}$ Support for this hypothesis from cohort studies, however, was lacking. ${ }^{4} \mathrm{~A}$ randomized clinical trial (RCT) is considered the most definitive study design to formally test a hypothesis, but RCTs also have substantial limitations that are often not fully recognized.

RCTs that produce the anticipated effect are easily interpreted; however, null RCTs are necessarily more difficult to interpret. In the WHI, issues of compliance to the intervention (few women reached the intervention target), timing in participant lifespan and duration of the intervention (all postmenopausal women, relatively short duration of intervention), the intervention itself (total fat versus specific fats), and ability to measure adherence to the intervention (based on self-report, which is notoriously unreliable for macronutrients $^{5}$ ) plague the interpretation of this trial. Null trials with known limitations cannot be assumed to necessarily 'trump' observational studies, but rather contribute to the totality of evidence.

In interpreting the $\mathrm{WHI}$ findings, some experts emphasize the $9 \%$ lower risk of breast cancer in the intervention group compared with the control group (albeit not statistically significant; $P=0.07$ ), and note that the benefit was both more dramatic (22\% lower risk) and statistically significant in women with the highest fat intake at baseline, which adds to the plausibility of a real effect. Others interpret the finding rigidly -no significant benefit was shown in the primary endpoint according to accepted criteria for statistical significance.

It is possible that, with additional follow-up, the observed benefit from the intervention will

\section{....moderating \\ dietary fat, especially animal fat \\ intake, is \\ unlikely to \\ produce any \\ adverse effects \\ and may \\ reduce breast \\ cancer risk}

ST Mayne is an Advisory Board member of Nature Clinical Practice Oncology.

\section{Competing interests}

The author declared she has no competing interests.

www.nature.com/clinicalpractice doi:10.1038/ncponc0518 become statistically significant. We are then put in the awkward position of contradicting ourselves, telling women that we were wrong in saying dietary fat doesn't matter (based on a $P$ value of 0.07 ) if in the future the benefit becomes statistically significant. The public is already confused about dietary recommendations. Could the investigators have simply extended the trial for an additional year to see if the beneficial trend continued? That course of action would have been preferred given the reduced power (approximately 60\%) of the trial when departures from the original design were considered, but with a trial of this visibility, involving this many women and this much expense, extension was impossible.

What do the WHI results hold for the future of nutritional epidemiology? We have learned that major dietary changes are difficult to implement, and, for dietary fat, even more difficult to verify. Even before the WHI findings, researchers had started to develop and validate objective indicators of dietary intake, with recent proposals for new biomarkers of total fat intake. ${ }^{6}$ Progress in the study of intake biomarkers is critical to better elucidate complex diet-disease relationships.

In the meantime, what should we tell women (including premenopausal women) about dietary fat and breast cancer? Despite a massive financial investment and a stellar team of investigators, definitive answers to this age-old question elude us. In the absence of scientific certainty, it seems most logical to consider the totality of the evidence and take a prudent approach. Accordingly, the statement that best conveys the scientific uncertainty is that moderating dietary fat, especially animal fat intake, ${ }^{7}$ is unlikely to produce any adverse effects and may reduce breast cancer risk. This is a complex answer to a seemingly simple question.

Supplementary information, in the form of a reference list, is available on the Nature Clinical Practice Oncology website. 